\title{
Itinéraires Itinéraires
}

Littérature, textes, cultures

2017-1 | 2018

Biographie et fiction

\section{Présentation}

\section{Françoise Palleau}

\section{(2) OpenEdition}

Journals

Édition électronique

URL : http://journals.openedition.org/itineraires/3659

DOI : $10.4000 /$ itineraires.3659

ISSN : 2427-920X

Éditeur

Pléiade

Référence électronique

Françoise Palleau, «Présentation », Itinéraires [En ligne], 2017-1 | 2018, mis en ligne le 15 février 2018, consulté le 24 septembre 2020. URL : http://journals.openedition.org/itineraires/3659; DOI : https:// doi.org/10.4000/itineraires.3659

Ce document a été généré automatiquement le 24 septembre 2020.

\section{(c) (i) (9)}

Itinéraires est mis à disposition selon les termes de la licence Creative Commons Attribution - Pas d'Utilisation Commerciale - Pas de Modification 4.0 International. 


\title{
Présentation
}

\author{
Françoise Palleau
}

1 Pour illustrer le lien entre la biographie et la fiction dans leur rapport à l'histoire, le présent numéro choisit pour exemple introductif le roman de Lily King, Euphoria (New York, Picador, 2014), qui contribue à éclairer un épisode de la vie de l'anthropologue américaine Margaret Mead. Cette dernière n'apparaît pas sous son vrai nom mais sous celui d'un personnage fictif, la fiction autorisant une exploration imaginaire plus riche que l'approche biographique strictement historique ne la permettait. Suivant cette trace, la littérature contemporaine permet-elle un renouveau de la biographie, et sous quelles modalités? Réciproquement, de quelle manière la biographie nourrit-elle encore la fiction, et permet-elle un effet de réel ou au contraire, une mise à distance plus ou moins importante du réalisme, ou encore, une nouvelle assise littéraire au récit historique ? Contrairement à l'idée reçue que la fiction serait l'ennemie de l'histoire et de la biographie historique, dans quelle mesure l'imaginaire fictif favorise-t-il l'empathie du lecteur de récit biographique historique? Autant de questions auxquelles plusieurs articles répondent, en considérant des biographies aussi diverses que celles de Tchekhov, ou bien de Spinoza et de Séraphine de Senlis conjointement, ou encore de Walter Pater, ou même les autofictions d'écrivaines vagabondes en quête d'identité au cours de leur cheminement.

2 Les réflexions de chercheurs eux-mêmes auteurs de récits de vie font partie intégrante de ce numéro, afin de rendre compte d'une pratique d'écriture et de la méthodologie qui la nourrit, la praxis ouvrant à des perspectives créatives, au sortir des sentiers théoriques ou strictement universitaires.

3 Ainsi, Rachel Blau DuPlessis, poète et universitaire américaine de renom, a-t-elle confié à Itinéraires un inédit sur son «itinéraire » autobiographique qui rassemble les deux aspects de sa carrière : un texte de réflexion discursive et philosophique accompagne des fragments autobiographiques de deux époques distinctes de sa vie, pour mettre en regard théorie et écriture de création d'une part, mais aussi deux époques différentes, l'histoire personnelle rejoignant ainsi la collective dans le poème diariste et dans la pensée qui fait retour sur le temps vécu. 
Elle utilise des signes typographiques pour indiquer cette relation critique, dont le signe de l'équation (=). Ainsi, elle montre sans le nommer son lien problématique avec un groupe de poètes américains des années 1960-1970, qui ont pratiqué ce qu'ils appellent la "poésie de la langue » ("language poetry»). Ces poètes mettaient l'accent sur les fonctions de la langue, d'ordre syntaxique et soumises à des contraintes quasioulipiennes par exemple, plutôt que sur la référence ou la thématique du contenu de leur production. La poésie n'est pas tant pour eux une question d'expression que de construction de la langue, une forme linguistique travaillée dans et par la langue. Si par bien des aspects le signe = fait entrer ces poètes en dialogue avec le texte publié ici, la réflexion engagée par l'auteur se démarque du groupe, auquel elle affirme ne pas appartenir, parce qu'elle a contribué à combler ce qu'elle estime leur défaut, au sens de manque : ils n'ont pas placé le féminisme au cœur de leurs préoccupations. Ils ont souvent favorisé la poésie en prose, sans narrativité, et intégré des formes réflexives et philosophiques dans leur production poétique. Comme pour leur répondre, Rachel Blau DuPlessis met ici les deux aspects en regard, en une dialectique entre la pratique de l'écriture de soi et le retour sur soi distancié par une réflexion théorisée. Mais ce groupe de poètes a souvent supprimé le genre de leur formulation et de leur pensée, ce qu'elle a souhaité replacer au centre de son questionnement de l'époque. L'autobiographie étant déjà un retour réflexif sur un soi construit, la spécularité du texte de Blau DuPlessis se double ici d'un retour sur des mouvements de pensée et d'art de son temps, incluant le féminisme et le post-structuralisme, ainsi que la question de la postmodernité éventuelle de son travail. Finalement, la réflexion intègre aussi des considérations socio-économiques et une critique sociale. La forme littéraire retrouve le monde dans et par sa syntaxe, dans les jeux de mots entre les «lignes» («lines») et les «liens » (ces derniers en français dans le texte original), confrontés tous deux aux mensonges politiques (« lies ») qui prétendent que la « ligne » ségrégative de la couleur de peau serait effacée dans une société post-raciale.

Outre ce texte à la fois autobiographique et biographique d'une génération, de sa politique et de ses mouvements littéraires, d'autres praticiens de la biographie ont contribué à ce numéro. Miriam Nichols présente son expérience de recherche sur le poète américain Robin Blaser (1925-2009), affilié à la Renaissance de Berkeley dans les années 1940 et 1950, proche des poètes Robert Duncan et Jack Spicer. Et enfin, l'écrivain contemporain américain Lou Rowan, co-directeur de ce numéro, revient sur sa pratique d'écriture biographique, qui l'a conduit à mener une enquête sur une figure de proue du monde de l'art à Seattle et sur son inclusion de fragments biographiques dans ses ouvrages de fiction.

Deux autres chercheurs étudient la manière dont un récit de vie peut en cacher un autre, dans le cas d'écrivains auteurs de biographies, à l'instar de Walter Pater, dans ses Vies imaginaires (Alexandre Bies), ou d'Irène Némirovsky, dans sa Vie de Tchekhov (Marta Cenedese). Un chercheur se penche sur la double mythification d'un film biographique, le «biopic » d'Eva Perón, figure politique légendaire à laquelle se surimpose l'image de l'interprète Madonna, rendant ainsi la mise en biographie complexe (Gabriel Laverdière).

7 Les auteurs de ce numéro ont souvent adopté des approches pluridisciplinaires, croisant deux ou plusieurs disciplines, telles narratologie, théorie de la réception et études cinématographiques, pour analyser l'autobiographie filmique de Woody Allen (Frédérique Brisset); histoire de la photographie et graphisme, pour cerner 
l'adaptation bédéiste de la biographie d'une photographe, Tina Modotti (Marie-Caroline Leroux) ; psychologie, études du genre et écriture de voyage, pour définir une catégorie peu explorée, celle de la "vagabonde » autobiographique (Dúnlaith Bird); histoire et autofiction, à propos de Doubrovsky (Anaïs Fusaro). Un auteur critique la réception normative et pathologisante de penseurs ou créateurs hors du commun, tels Spinoza et Séraphine de Senlis (Kisito Hona).

8 En dernier lieu et pour conclure, l'analyse des frontières parfois ténues ou même indiscernables entre la biographie, l'autobiographie et l'autofiction permet en outre d'éclairer les approches des « récits de vie », «biofiction » ou des récits " mémoristes » fictifs ou authentifiés. Dans cet objectif, l'article de Miruna Craciunescu offre une présentation large et récapitulative de l'état de l'art théorique, en prenant position sur les évolutions les plus récentes. Enfin, l'article de Khalid Lyamlahy analyse le discours et le « biographème » barthien, pour caractériser les stratégies d'une biographie fictive (par l'écrivain mexicain Alain-Paul Mallard), qui mystifie l'authentification biographique, pour le plaisir du jeu fictionnel et de l'écriture de la jouissance.

Le choix d'élargir les études sur la biographie et la fiction à des aires linguistiques variées, qu'elles soient anglophones ou hispanophones, dans des articles en français ou en anglais, et à des médias artistiques aussi différents que la littérature, le cinéma et la bande dessinée, a pour objectif d'apporter une contribution au souci de décloisonnement intellectuel et culturel des études biographiques actuelles, comme en témoignent les travaux de nos collègues de l'université de Groningen dans leur récente publication panoramique sur la place de la biographie dans les humanités, The Biographical Turn ${ }^{1}$. Par "littérature ", s'entend ici l'écriture littéraire au sens large, incluant la poésie, la prose poétique et les œuvres intermédiales telles que l'adaptation d'une biographie en bande dessinée.

\section{NOTES}

1. Hans Renders, Binne de Haan et Jonne Harmsma (dir.), The Biographical Turn: Lives in History, Londres, Routledge, 2017.

\section{AUTEUR}

\section{FRANÇOISE PALLEAU}

Université Paris 13, Pléiade (EA 7338) 